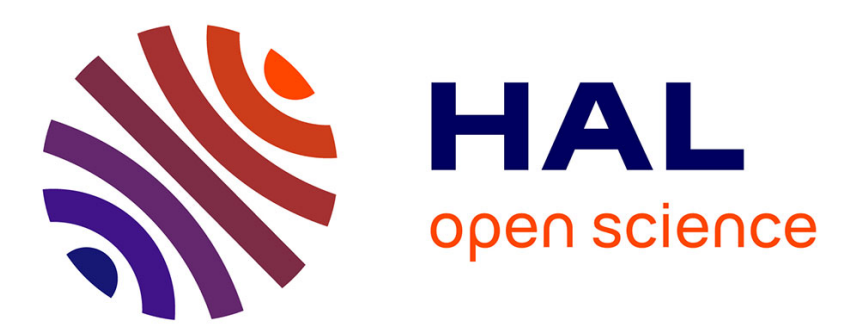

\title{
Croissance et développement au champ de l'igname (Dioscorea alata L.) à partir de plants produits par culture in vitro
}

André Lacointe, Claude Zinsou

\section{- To cite this version:}

André Lacointe, Claude Zinsou. Croissance et développement au champ de l'igname (Dioscorea alata L.) à partir de plants produits par culture in vitro. Agronomie, 1987, 7 (5), pp.331-338. hal-00884998

\section{HAL Id: hal-00884998 \\ https://hal.science/hal-00884998}

Submitted on 1 Jan 1987

HAL is a multi-disciplinary open access archive for the deposit and dissemination of scientific research documents, whether they are published or not. The documents may come from teaching and research institutions in France or abroad, or from public or private research centers.
L'archive ouverte pluridisciplinaire HAL, est destinée au dépôt et à la diffusion de documents scientifiques de niveau recherche, publiés ou non, émanant des établissements d'enseignement et de recherche français ou étrangers, des laboratoires publics ou privés. 


\title{
Croissance et développement au champ de l'igname (Dioscorea alata L.) à partir de plants produits par culture in vitro
}

\author{
André LACOINTE $\left({ }^{*}\right)$ \& Claude ZINSOU \\ I.N.R.A., Laboratoire de Physiologie et Biochimie végétales, Centre de Recherches des Antilles et de la \\ Guyane, B.P. 1232, F 97184 Pointe-à-Pitre Cedex, Guadeloupe
}

\begin{abstract}
La culture in vitro des ignames Dioscorea alata $\mathrm{L}$. produit des plantes aptes à croître en plein champ après passage par 2 milieux d'adaptation intermédiaires. La morphogenèse initiale de ces vitroplants les distingue du matériel traditionnel obtenu par fragmentation des tubercules, et les apparente au contraire aux jeunes semis d'espèces voisines. Ultérieurement, ces particularités juvéniles sont masquées par l'émergence de grandes lianes semblables à celles du matériel usuel. Comme en culture traditionnelle, la partie aérienne est d'abord seule (avec les racines) à crô̂tre pendant plusieurs mois. La mise en réserve dans les tubercules, différenciés pourtant très précocement, ne devient significative qu'après diminution de l'intensité de croissance aérienne. Le nombre et la morphologie des tubercules en fin de cycle sont comparables à ce que l'on observe sur le matériel conventionnel ; mais, dans nos conditions expérimentales, leur poids est inférieur de moitié. On peut les récolter plusieurs semaines avant la disparition totale de l'appareil aérien sans conséquence sur le rendement ni sur le comportement ultérieur des tubercules.

Les corrélations entre poids de matière sèche des tubercules et plusieurs paramètres caractérisant le développement de la partie aérienne sont utilisables pour une aide à la prévision des rendements et pour une évaluation de l'efficacité de la plante à convertir l'énergie lumineuse incidente en amidon stocké dans le tubercule.
\end{abstract}

Mots clés additionnels : Tubercule, tubérisation, cycle biologique, agronomie tropicale.

Field growth and development of yam (Dioscorea alata) plantlets from in vitro culture.

Plantlets of yam (Dioscorea alata L.) from in vitro culture were adapted to field conditions by culturing in two successive, transition media. After planting in April, the early growth pattern of these plantlets was different from that of traditional sets obtained by breaking up tubers, and resembled instead that of young seedlings of related yam species. Later, these juvenile-looking characteristics vanished with the emergence of long vines identical to those of normal yam plants. As observed in normal cultivation, tubers, though present soon after planting, did not grow significantly before August, when the growth rate of the aerial parts fell. At the end of the cycle, the number and shape of tubers was the same as for conventional plants, but their weight was halved (in our experimental conditions). Tubers could be harvested several weeks before vine drying without any consequences for yield or for the subsequent rest period. The correlations between tuber dry matter weight and several parameters which characterize development of the aerial parts could be used to forecast yield and to evaluate the plant's ef ficiency in converting incident light into tuber starch.

Additional key words : Tuber, tuberization, biological cycle, tropical agronomy.

\section{INTRODUCTION}

Les espèces alimentaires d'ignames (Dioscorea spp.) ne produisent pas de graines viables, sauf $D$. trifida et parfois $D$. cayenensis-rotundata; les jeunes semis étant alors très hétérogènes (DEGRAS et al., 1977a).

$\left({ }^{*}\right)$ Adresse actuelle : I.N.R.A., Laboratoire de Bioclimatologie, Domaine de Crouelle, F 63039 Clermont-Ferrand Cedex.
La propagation des ignames s'effectue donc par voie végétative, généralement par fractionnement des tubercules ou au moyen de bulbilles pour certaines espèces. La multiplication occasionne ainsi le prélèvement d'un pourcentage non négligeable de la récolte et la levée est en outre souvent hétérogène (MIEGE, 1957 ; Trouslot, 1978). D'autres méthodes ont été expérimentées, comme le bouturage (AHOUsSOU et al., 1980), ou le sevrage des jeunes pousses issues du tubercule (MATHURIN, 1983). Outre la variabilité 
parfois plus importante induite par ces méthodes (AHOUSSOU et al., 1980), les travaux qu'elles impliquent ne les rendent intéressantes que pour de petites exploitations (MATHURIN, 1983).

Dans ce contexte, la multiplication in vitro peut présenter a priori de nombreux avantages : taux de multiplication inégalé permettant une production à très grande échelle occupant peu de place, coût réduit par plante produite et (dans certaines conditions excluant notamment la callogenèse) variabilité diminuée.

Les premiers essais de culture et de multiplication d'ignames in vitro remontent à une dizaine d'années (MASCARENHAS et al., 1976 ; CHATURVEDI, 1975), mais ils concernaient exclusivement des espèces intéressantes pour leur teneur en stéroïdes, sans intérêt alimentaire, comme $D$. floribunda ou $D$. deltoidea.

A ce jour, le nombre d'études publiées sur la multiplication de l'igname in vitro reste très limité, et peu d'entre elles portent sur le comportement ultérieur des plantules au champ (MANTELL et HAQUE, 1979; ARNOLIN et DEGRAS, 1983). Cet aspect est pourtant fondamental si l'on envisage une application agronomique de cette technique.

Le travail que nous allons présenter constitue une première approche de la question. S'il ne permet pas une extrapolation à grande échelle, il permet cependant une description assez fine du mode de croissance et de développement des plantes issues de culture in vitro (vitroplants). Nous insisterons sur les similitudes, mais aussi sur les particularités que présente ce matériel vis-à-vis du matériel traditionnel provenant de la germination d'un fragment de tubercule, matériel assez bien connu (CHAPMAN, 1965 ; SOBULO, 1972 ; DEGRAS $e t$ al., $1977 b$ et $c$; TROUSLOT, 1978 ; CLAIRON \& ZINSOU, 1980 ; OKOLI, 1980 ; TROUSLOT, 1985).

\section{MATÉRIEL ET MÉTHODES}

\section{A. Matériel végétal}

La culture in vitro a été amorcée à partir de boutures uninodales (limbe foliaire supprimé) de lianes de D. alata cv. «Lupias» stérilisées à l'hypochlorite de calcium 7 p. 100, puis entretenue par prélèvement périodique de boutures uninodales sur des plantules du cycle précédent. Le milieu de culture, mis au point par MARTIN et ses collaborateurs à l'I.N.R.A. de Dijon, contient les éléments de base suivants :

- les macro- et microéléments de Murashige \& SKOOG (1962) ;

- les vitamines de MOREL \& MARTIN (1965) ;

- saccharose à $30 \mathrm{~g} / 1$; myo-inositol à $100 \mathrm{mg} / 1$;

- L-glutamine à $200 \mathrm{mg} / 1$; chlorhydrate de cystéine à $1 \mathrm{mg} / \mathrm{l}$;

- charbon actif à $20 \mathrm{~g} / \mathrm{l}$; agar-agar à $7 \mathrm{~g} / \mathrm{l}$.

La température est de $25^{\circ} \mathrm{C} \pm 1{ }^{\circ} \mathrm{C}$ et la photophase de $12 \mathrm{~h}$. L'éclairement (puissance électrique comprise entre 100 et 150 Watts par $\mathrm{m}^{2}$ ) est assuré par des tubes fluorescents «Mazdafluor, lumière du jour » de $40 \mathrm{~W}$.

Après 3 mois de culture in vitro, le développement caulinaire et racinaire des plantules permet soit de débuter un nouveau cycle de multiplication in vitro, soit, en vue de la plantation au champ, de les repiquer sur un substrat de tourbe. On élimine les plantules n'ayant pas eu une croissance normale en tube ; les autres sont éventuellement divisées pour ne conserver qu'une pousse par plantule (fig. 1). Elles sont alors placées sous enceinte transparente à brumisation périodique, protégée des rayons solaires directs. De $6 \mathrm{~h}$ à $18 \mathrm{~h}$, l'éclairement naturel est supplémenté par 2 tubes fluorescents de $40 \mathrm{~W}$ pour $1 \mathrm{~m}^{2}$.

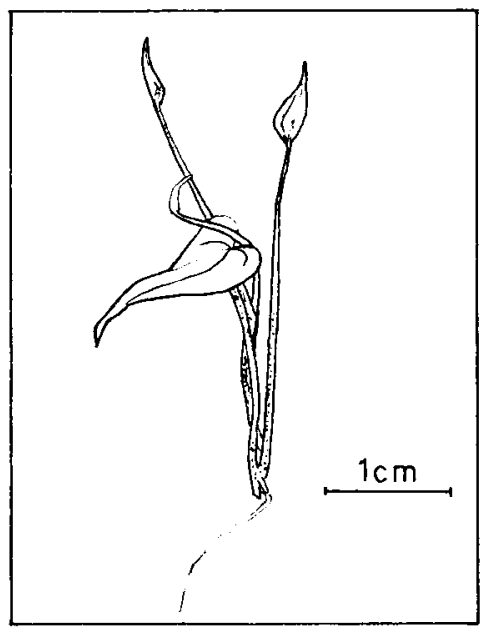

Figure 1

Aspect d'une plantule à la sortie du tube, avant repiquage sur un substrat de tourbe.

A plantlet just taken up from the culture medium, before planting into the first transition medium (peaty soil under misting).

Un mois plus tard, les plantules sont placées en pots emplis de terre, et transférées en serre sans brumisation ni éclairage supplémentaire. La plantation au champ a lieu quelques semaines plus tard, avec un taux de reprise de 95 à 100 p. 100 .

Ce protocole fournit des jeunes plants à toute époque de l'année. Cependant, afin de comparer dans des conditions équivalentes leur développement avec celui des plantes issues de fragments de tubercules, nous avons réalisé la plantation en avril (22/04/83), époque de germination de ce matériel en conditions naturelles.

\section{B. Conditions de culture au champ}

La culture est conduite sur un sol ferralitique de la Basse-Terre (Guadeloupe), en 6 parcelles de 20 plantes espacées de $1 \mathrm{~m}$ en tous sens. L'engrais $(\mathrm{N} / \mathrm{P} / \mathrm{K}$ : $120 / 70 / 120$, respectivement sous forme de $\mathrm{NH}_{4} \mathrm{NO}_{3}$, supertriple et $\mathrm{K}_{2} \mathrm{SO}_{4}$ ) est apporté une semaine avant plantation. En période de sécheresse, on irrigue par aspersion. Les plantes sont tuteurées 1 mois après plantation, et traitées contre l'anthracnose par pulvérisation foliaire d'un mélange de «Daconil » et «Benlate ».

\section{Mesures et prélèvements}

Afin de définir un "état initial » de la plantation aussi précisément que possible, on a effectué, le 10 mai, sur tous les individus, les relevés non- 
destructifs des paramètres figurant au tableau 1 . Simultanément, on a arraché 10 d'entre eux, donnant la morphologie de la plante entière.

De même, la croissance et le développement ultérieurs ont été suivis de 2 façons complémentaires :

1. Des prélèvements mensuels de 7 à 9 plantes (plus une quarantaine à la récolte) ont permis une description de la morphogenèse des tubercules à partir des prétubercules, structures complexes propres au genre Dioscorea (MIEGE, 1952, cité par DEGRAS, 1978). A chaque prélèvement, un certain nombre de tubercules sont stockés en clayettes pour noter la date de germination; les autres sont séchés à $105^{\circ} \mathrm{C}$ pendant 3 jours en étuve ventilée pour déterminer le poids de matière sèche. Sur la partie aérienne on note le nombre et la longueur des tiges ainsi que les nombres de feuilles adultes (image de la croissance instantanée à la date $\mathrm{t}$ ). On détermine également le poids de matière sèche des tiges et des feuilles. La surface foliaire est mesurée à l'aide d'un planimètre optique «Metraplan ».

2. Sur 9 plantes choisies en début de culture, on a relevé mensuellement les nombres de feuilles adultes et de feuilles en croissance ainsi que la longueur de chaque tige. La surface foliaire est estimée, à 20 p. 100 près, par régression multiple à partir de 4 paramètres mesurés sur une feuille représentative de chaque tige (LACOINTE, 1984). On en déduit, pour chaque plante, un «indice de potentialité photosynthétique " intégré sur toute la saison : $i=\sum \mathrm{S}_{i} \mathrm{R}_{i}(\Delta \mathrm{t})_{i}$, où $\mathrm{S}_{i}$ et $\mathrm{R}_{i}$ sont respectivement la surface foliaire et le rayonnement global journaliers moyens pendant l'intervalle $(\Delta \mathrm{t}) i$ séparant 2 mesures.

On note enfin la date d'apparition des tiges nouvelles, estimée à quelques jours près en fonction de leur longueur et de l'aspect de leurs feuilles, et leur position dans l'architecture de la plante. A la chute du feuillage les tubercules sont récoltés et traités comme en (1).

Pour les paramètres comparables, les courbes issues des prélèvements mensuels (1) sont a priori plus représentatives du niveau moyen de l'ensemble de la population que les courbes moyennes des suivis individuels (2) ; cependant, cette opinion est infirmée $a$ posteriori par la bonne coïncidence des deux types de courbes. Comme la forme des courbes (2) est par ailleurs exempte de variabilité individuelle d'un point à l'autre, nous nous limiterons à la présentation de ces dernières.

\section{Méthodes statistiques}

Les barres verticales figurant sur certaines courbes représentent l'intervalle de confiance au niveau $\alpha=0,05$, calculé par la méthode de Student. L'égalité des dates de germination de 2 populations de tubercules est testée au niveau $\alpha=0,05$ par la méthode non-paramétrique de Mann et Whitney. Pour tester l'augmentation du coefficient de détermination liée à l'adjonction d'une variable explicative supplémentaire en régression linéaire, on teste la signification du coefficient de corrélation partielle correspondant, au niveau $\alpha=0,05$.

Ces méthodes sont exposées dans DAGNELIE (1975a et $b$ ).

\section{RÉSULTATS}

\section{A. Croissance et développement des vitroplants}

\section{Morphologie des vitroplants 20 jours après plan- tation}

Malgré une grande variabilité (tabl. 1) que l'on pourrait probablement restreindre par une sélection plus sévère au cours des repiquages in vitro, les caractères morphologiques essentiels des plantules sont semblables (fig. 2).

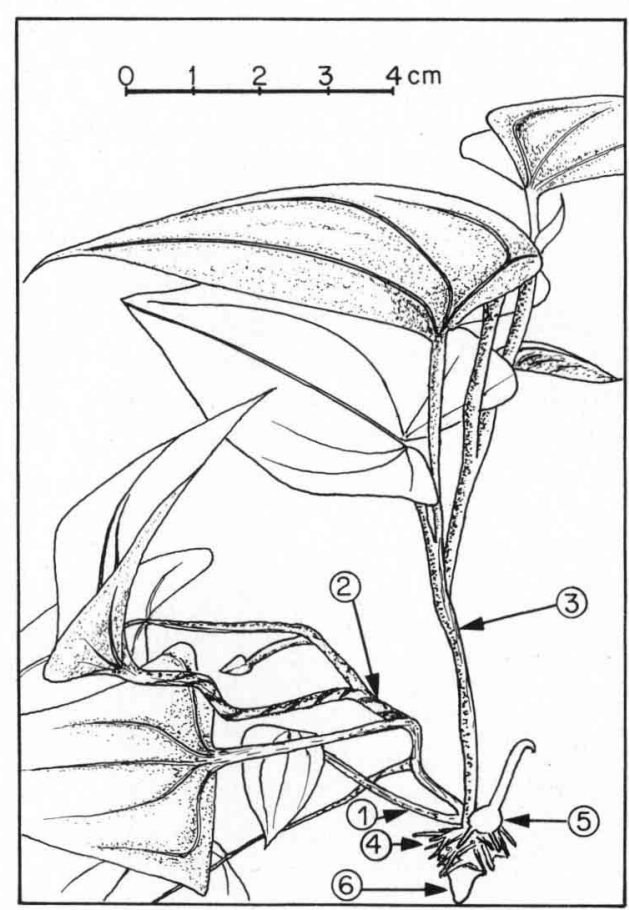

Figure 2

Aspect d'un vitroplant arraché le 10 mai, 18 jours après plantation au champ.

(1) tige initiale, développée in vitro

(2) tige de "génération 2 », développée en godet de tourbe;

(3) tige de "génération 3 ", développée en pot

(4) ensemble de prétubercules peu distincts les uns des autres, por-

tant de nombreuses racines coronaires (sectionnées sur ce dessin);

(5) prétubercule basal d'une nouvelle pousse avec racines coronai-

res naissantes

(6) tubercule sensu stricto.

A set dug up on May 10th, 18 days after planting.

(1) earliest stem, grown in vitro:

(2) " 2nd generation " stem, grown in the first transition medium (with misting);

(3) "3rd generation" stem, grown in the second transition medium (greenhouse);

(4) set of aggregated primary nodal complexes bearing many crown shoots (cut off on this drawing);

(5) primary nodal complex of a new shoot, emitting crown roots;

(6) tuber sensu stricto.

On distingue trois «générations » de tiges, de diamètre et de longueur croissants, développées respectivement en tube, en godet de tourbe et en pot. Non volubiles, elles sont généralement en forme de «zigzag » dont les angles sont en position nodale.

Outre les racines, la partie souterraine est constituée essentiellement de prétubercules attenant les uns aux 
TABLEAU 1

Caractéristiques (moyennes \pm écarts-types) des vitroplants, mesurées le 10/05/83, sur (a) 103 ou (b) 9 individus. (*) Seules les tiges de longueur supérieure à $3 \mathrm{~cm}$ ont été recensées.

Biometric characteristics (average \pm standard deviation), as measured on May I0th on (a) 103 or (b) 9 individuals. (*) Stems shorter than $3 \mathrm{~cm}$ were not taken into account.

(a)

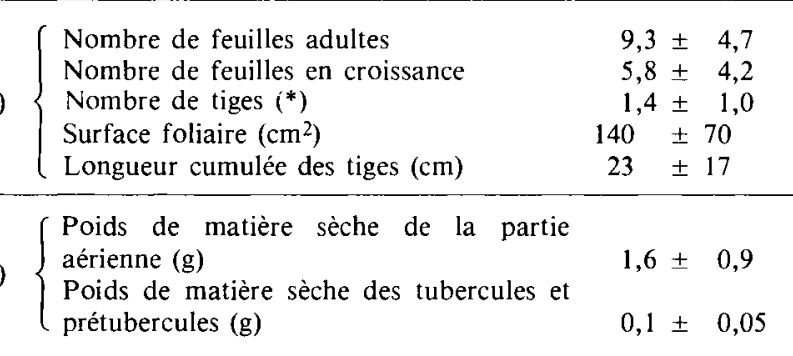

autres. Ce sont des structures globuleuses de quelques millimètres, portant la quasi-totalité des racines disposées en couronne. Tout rameau porte un prétubercule à sa base, si celle-ci est enterrée. La succession des " générations " de tiges apparaît liée à une filiation de leurs prétubercules basaux (fig. 3).

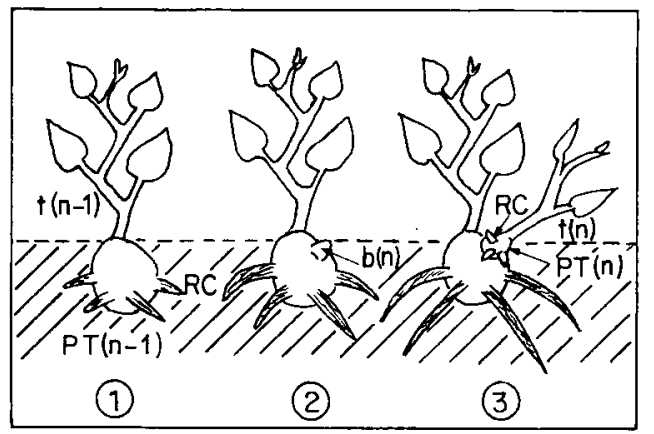

Figure 3

Schéma de « filiation 》 des prétubercules.

$t(n-l)$ et $t(n):$ tiges de "générations » $n-l$ et $n$;

$P T(n-1)$ et $P T(n)$ : prétubercules basaux de $t(n-I)$ et $t(n)$;

$b(n)$ : bourgeon-« fils », différencié à partir de $P T(n-1)$;

$R C$ : racines coronaires.

Successive « generations » of primary nodal complexes. $t(n-l), t(n):$ stems of « generations » $n-1$ and $n$ $P T(n-I), P T(n)$ : primary nodal complexes of $t(n-1), t(n)$; $b(n):$ : daughter »-bud, differentiated from $P T(n-1)$; $R C$ : crown roots.

Certains prétubercules ( 1 à 2 par plante, généralement les plus anciens) ont déjà différencié un tubercule sensu stricto qui se distingue nettement par la couleur (ocre-rosé), le géotropisme positif et l'absence quasi-totale de racines.

\section{Croissance et développement ultérieurs}

\section{a) La partie aérienne}

Il apparaît de nouvelles tiges jusqu'au début du mois d'octobre, avec une fréquence non uniforme au cours du temps : pour chaque plante la fréquence suit une répartition multimodale (fig. 4). Les pics de fréquence (4 à 7 suivant les individus) s'étalent chacun

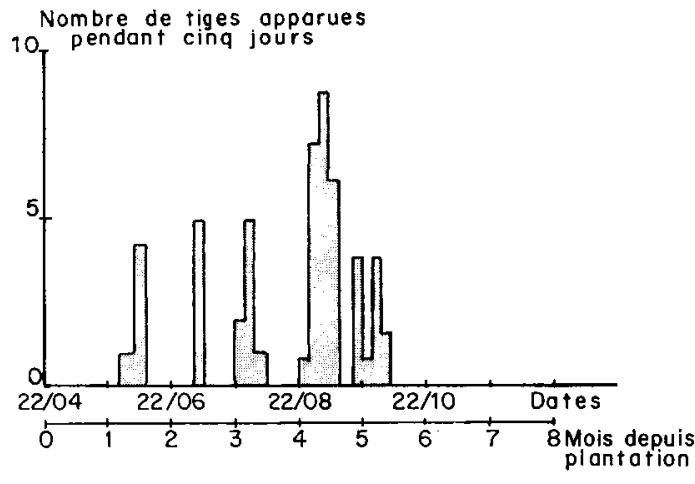

Figure 4

Fréquence d'apparition de nouvelles tiges sur un individu pendant toute la saison.

Variation in number of new shoots emitted during 5 days by an individual plant.

sur une dizaine de jours; cependant, alors que les premiers sont nettement séparés, les suivants ont tendance à se recouvrir. Ils ne sont pas synchronisés d'un individu à l'autre. Une nouvelle série de tiges apparaît lorsque la majorité des tiges de la série précédente ont atteint leur longueur maximale.

$\mathrm{Au}$ cours du temps, on assiste à un déplacement du lieu préférentiel de croissance des nouvelles tiges ainsi qu'à une modification de leur longueur (fig. 5). Cette évolution concerne également la morphologie (lianes ou rameaux rectilignes) et la phyllotaxie : alterne sur la partie inférieure des grandes lianes issues de la base de la plante, opposée-décussée ailleurs.

Après l'arrêt de sa croissance (accompagné généralement de la nécrose ou de la chute des derniers entrenœuds), une tige donnée ne survit que 3 mois environ ; ensuite elle se nécrose à partir de l'apex. En conséquence, la naissance et la croissance en longueur des nouvelles pousses d'une part et la sénescence et la nécrose des tiges âgées d'autre part, s'observent simultanément sur la même plante pendant 2 à 3 mois. La nécrose se généralise à l'ensemble du système aérien à partir de la mi-novembre, de façon assez simultanée pour tous les individus puisque la proportion de vitroplants complètement défoliés passe de 0 , au $1^{\text {er }}$ décem-

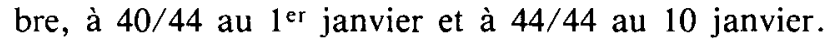

Cette croissance continue de la partie aérienne jusque peu avant sa chute se traduit par un développement maximal début octobre (fig. 6), suivi immédiatement d'un déclin rapide.

Des inflorescences (mâles) ont été observées sur 14 plantes parmi 57 , entre mi-octobre et minovembre. Le fait qu'une plante ait fleuri ou non n'a aucune incidence sur la date de nécrose complète de la partie aérienne.

\section{b) La partie souterraine}

Jusque vers juillet, chaque nouvelle tige issue du collet porte un prétubercule à sa base. Le développement de la partie souterraine (racines exceptées) reste très limité (fig. 7).

A partir de juillet, les nouvelles tiges apparaissant au collet ne présentent plus systématiquement de renflement à leur base. Cependant, la masse prétuberculaire (ainsi nommée parce qu'on distingue de moins en moins les prétubercules qui la composent) peut encore 


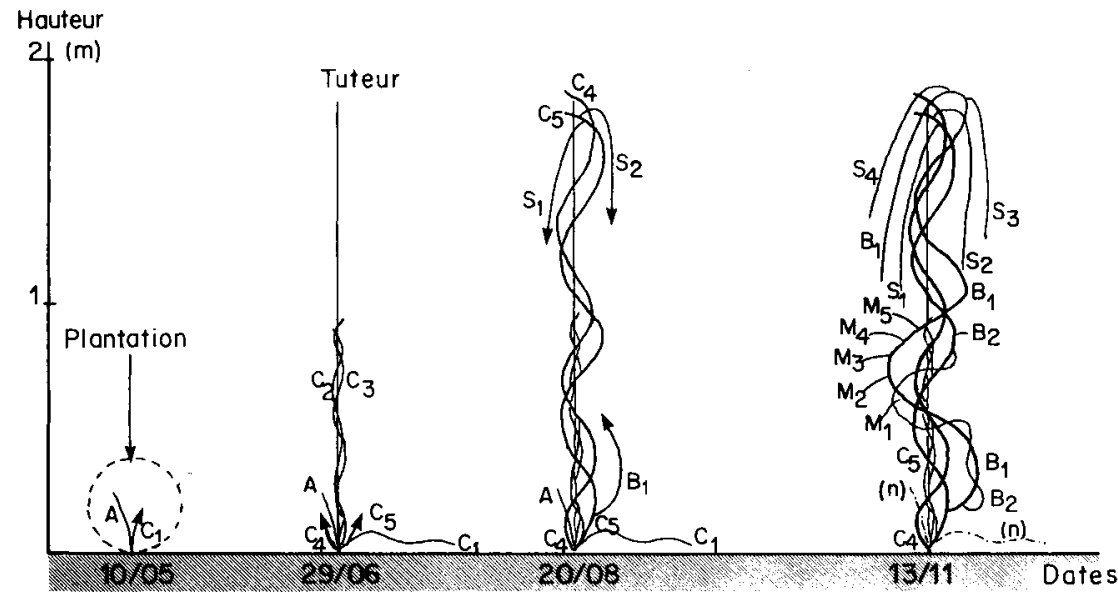

Figure 5

Evolution de l'architecture des vitroplants d'ignames pendant la phase végétative len réalité on compte environ 2 fois plus de tiges qu'il n'en figure sur ce schéma simplifié).

$A$ : tige antérieure à la plantation;

$C_{1}$ à $C_{5}$ : tiges issues du collet (généralement volubiles);

$B_{1}$ et $B_{2}$ : lianes issues de bourgeons axillaires de la région basale des axes $C_{i}$;

$M_{1}$ à $M_{5}$ : rameaux axillaires courts issus de la région médiane des axes $C_{i}$ et $B_{i}$;

$S_{1} \grave{a} S_{4}$ : rameaux axillaires pendants issus de la région sommitale des axes $C_{i}$ et $B_{i}$;

(n) : tige nécrosée.

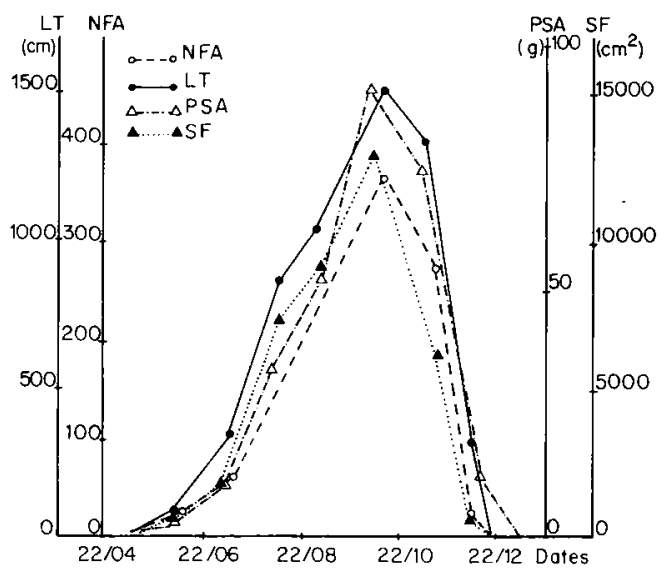

Figure 6

Croissance de la partie aérienne des vitroplants.

NFA : nombre de feuilles adultes;

$L T$ : longueur cumulée des tiges;

PSA : poids de matière sèche de la partie aériene;

$S F \quad$ : surface foliaire totale.

Moyennes (sauf PSA) des suivis individuels de 9 plantes. Coefficients de variation avoisinant $60 \mathrm{p} .100$, sans incidence sur la forme des courbes (cf. II.C).

Growth of the aerial part.

NFA : number of full-grown leaves;

$L T:$ total vine length;

PSA : dry matter weight of aerial parts;

$S F:$ total leaf area.

Averages of non-destructive (except for PSA), monthly measurements on 9 plants. Coefficients of variation are about $60 \%$.

grossir de façon isotrope jusqu'au diamètre de 2 à $3 \mathrm{~cm}$, tout en se lignifiant et en se subérifiant. La formation de nouveaux tubercules à partir de quelques prétubercules est possible jusque vers août (mais est exceptionnelle sur les prétubercules postérieurs à juin).
Development of plant architecture (the actual number of stems is about twice than shown).

$A$ : stem grown prior to planting.

$C_{T} C_{5}$ : stems growing from the collar (generally vines);

$B_{1}-B_{2}$ : vines from axillary buds of the lower part of stems $C_{i}$,

$M_{T}-M_{5}$ : short axillary shoots from the middle part of stems $B_{i}$ and $C_{i}$;

$S_{r} S_{4}$ : hanging axillary shoots from the upper part of stems $B_{i}$ and $C_{i}$;

(n) : dry stem

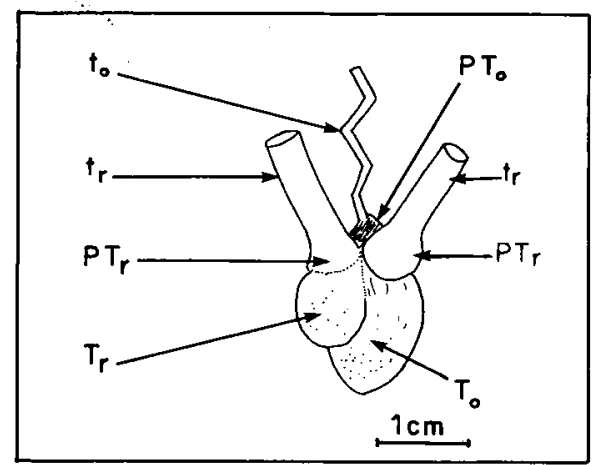

Figure 7

Partie souterraine (racines supprimées) d'une plante prélevée le 11/07/83.

$t_{0}:$ tige antérieure à la plantation;

$t_{r}:$ tige récente à prétubercule bien individualisé ;

$P T_{0}$ : prétubercule de la base de $t_{0}$

$P T_{r}$ : prétubercule de la base de $t_{r}$;

$T_{0}$ : tubercule issu de $P T_{0}$;

$T_{r}$ tubercule issu de $P T_{r}$

Underground part (cleared of roots) on July 11th.

$t_{0}:$ stem grown prior to planting;

$t_{r}$ : young shoot with a clearly differentiated primary nodal complex :

$P T_{0}, P T_{r}$ : primary nodal complexes of $t_{0}, t_{r}$;

$T_{0}, T_{r}$ : tubers differentiated from $P T_{0}, P T_{r}$.

Très lente jusqu'alors, la croissance pondérale des tubercules (fig. 8) s'accélère au début du mois d'août pour ne cesser qu'en novembre, au cours de la sénescence avancée du système aérien. L'arrêt de la croissance en poids s'accompagne de la stabilisation de la teneur en matière sèche, en augmentation jusqu'alors, autour de 33 p. 100

A la récolte, début janvier, les prétubercules sont généralement fondus en une seule masse ("tête») prolongée par 2 à 3 tubercules (fig. 9). 


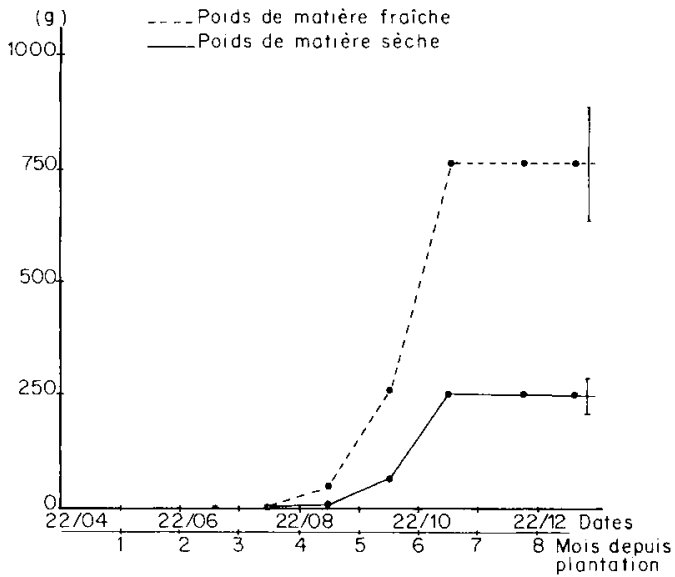

Figure 8

Evolution pondérale de la partie souterraine (sans les racines). Variation in underground part weight (cleared of roots). -.--- Fresh matter weight.

- Dry matter weight.

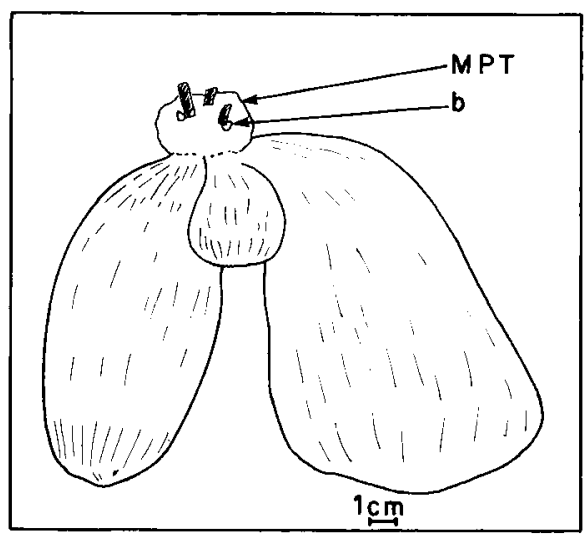

Figure 9

Partie souterraine d'une plante à la récolte (8/01/1984).

MPT : masse prétuberculaire $(=$ " tête ») portant des restes de tiges nécrosées:

$b:$ bourgeon entouré d'écailles rigides.

Underground part at harvest (January 8th).

MPT: " head" resulting from the confluence of several primary nodal complexes;

$b:$ bud with scales.

En stockage traditionnel sur clayettes ventilées, la germination des tubercules se produit entre le 10 avril et le $1^{\text {er }}$ juin, soit 4 à 5 mois après la fin de la phase feuillée.

\section{B. Relation entre développement de la partie aérienne pendant la phase feuillée et poids des tubercules à la récolte}

L'étude de cette relation aide à la prévision du rendement en poids de tubercules, et fournit des indications sur le rendement énergétique de l'ensemble des processus allant de l'interception de l'énergie lumineuse à son stockage sous forme chimique dans un organe spécialisé.

Cette étude, limitée au modèle linéaire, a conduit à la distinction entre 2 types de paramètres caractérisant le développement de la partie aérienne :
1. Des paramètres «statiques» relatifs à la taille maximale de la partie aérienne : surface foliaire (SF), longueur cumulée des axes (LT), nombre de feuilles adultes (NFA). Ils contiennent à peu près tous la même information (il suffit donc d'en mesurer un seul) sur le poids de matière sèche du tubercule (PS), quantifiable par le coefficient de détermination de la régression linéaire de $P S$ sur le paramètre considéré : $\mathrm{R}^{2} \simeq 0,84$ pour 9 plantes. Ces régressions donnent une estimation aisée du rendement final dès le début du mois d'octobre, soit 3 mois avant la récolte. Ainsi,

$$
\mathrm{PS}_{\text {g. de Mat. sc̀che }}=28+0,016 \times \mathrm{SF}_{\mathrm{cm}^{2}} \quad(\mathrm{r}=0,92)
$$

2. Un paramètre «dynamique » intégrant l'évolution de la partie aérienne sur toute la saison: l'« indice de potentialité photosynthétique » (IPP) défini plus haut. Son obtention est matériellement plus lourde que celle des paramètres précédents mais il contient plus d'information sur PS $\left(R^{2}=0,91\right)$; en outre, celle-ci est de nature différente puisque $\mathrm{R}^{2}=0,98$ (significativement $>0,91$ ) pour la régression double de PS sur IPP et l'un des paramètres LT, SF, NFA. Par ailleurs, la droite de régression $P S=f(I P P)$ passe par l'origine ; sa pente est donc un coefficient de proportionnalité directe : $0,11 \mathrm{~g}$ de matière sèche/M J d'IPP. Un calcul grossier assimilant la forme de la partie aérienne à une colonne cylindrique, tenant compte des composantes diffuse et directe du rayonnement global et de la trajectoire du soleil permet d'estimer qu'une valeur de $1 \mathrm{MJ}$ pour l'IPP correspond à $0,45 \mathrm{MJ}$ de rayonnement incident sur le feuillage. Le rendement énergétique (en matière sèche stockée dans le tubercule, constituée à 90 p. 100 d'amidon) est alors de $0,25 \mathrm{~g} / \mathrm{MJ}$ de rayonnement incident.

\section{DISCUSSION ET CONCLUSIONS}

\section{A. Comparaison des vitroplants avec d'autres types de matériel végétal}

En début de culture, nos vitroplants se distinguent nettement du matériel traditionnel par leur architecture, plus complexe, et par la morphologie de leurs tiges. En effet, chez les plants provenant de fragments de tubercules sur le même cultivar (CLAIRON \& ZiNSOU, 1980), les premiers axes ( 2 simultanés en moyenne) sont d'emblée de puissantes lianes dont les prétubercules basaux ne produisent pas d'axes supplémentaires.

En revanche, les vitroplants peuvent être rapprochés des jeunes semis d'espèces voisines. Chez $D$. trifida (DEGRAS et al., 1977a) et D. rotundata (SADIK \& OKEREKE, 1975 ; TROUSLOT, 1985), le ou les quelques axes présents pendant les 2 ou 3 premiers mois suivant la germination sont courts et non volubiles : leur morphologie en «zig-zag » (SADIK \& OKEREKE, 1975) est semblable à celle que présente la figure 2 . Les plantules de ces 2 espèces, ainsi que de quelques autres (LAWTON \& LAWTON, 1969), développent au cours de la saison des axes latéraux se succédant au niveau du collet (hypocotyle), qui se renfle en prétubercule plus ou moins précocement. 
Ces similitudes morphologiques et morphogénétiques entre jeunes semis et vitroplants confèrent à ces derniers un caractère juvénile.

Des axes volubiles apparaissent au cours du développement ultérieur des vitroplants. L'aspect général de ces derniers, dominé par les grandes lianes, finit par devenir assez semblable à celui des individus issus de tubercules-mères, mais leur architecture fine reste plus complexe. C'est également ce que l'on observe pour les semis d'espèces voisines (DEGRAs et al., 1977a, TrousLot, 1985).

De même, la complexité de la partie souterraine des vitroplants a tendance à s'estomper au cours de la saison puisque, pour 4 prétubercules formés en moyenne, on obtient seulement 2,5 tubercules à la récolte, soit le même résultat que sur le matériel traditionnel qui ne comporte que 2 prétubercules (tabl. 2).

Les dimensions finales atteintes par les organes des vitroplants sont inférieures à celles observées sur le matériel traditionnel (tabl. 2) ; DEGRAS et al. (1977a) ont fait la même constatation pour les semis de $D$. trifida. En revanche, les dynamiques respectives de croissance de la partie aérienne et des tubercules (fig. 10) sont les mêmes que pour le matériel traditionnel (CHAPMAN, 1965 ; SOBULO, 1972 ; DEGRAS et al., $1977 b$ et $c$; TROUSLOT, 1978 et 1985 ; OKOLI, 1980 ; CLAIRON \& ZINSOU, 1980) : la croissance du tubercule ne devient appréciable qu'après un ralentissement sensible de la croissance de la partie aérienne, indiqué par la diminution du nombre de feuilles en croissance après un maximum en août.
Il n'y a pas non plus de différences entre les 2 types de matériel pour le délai de germination du tubercule après récolte et la morphologie des germes.

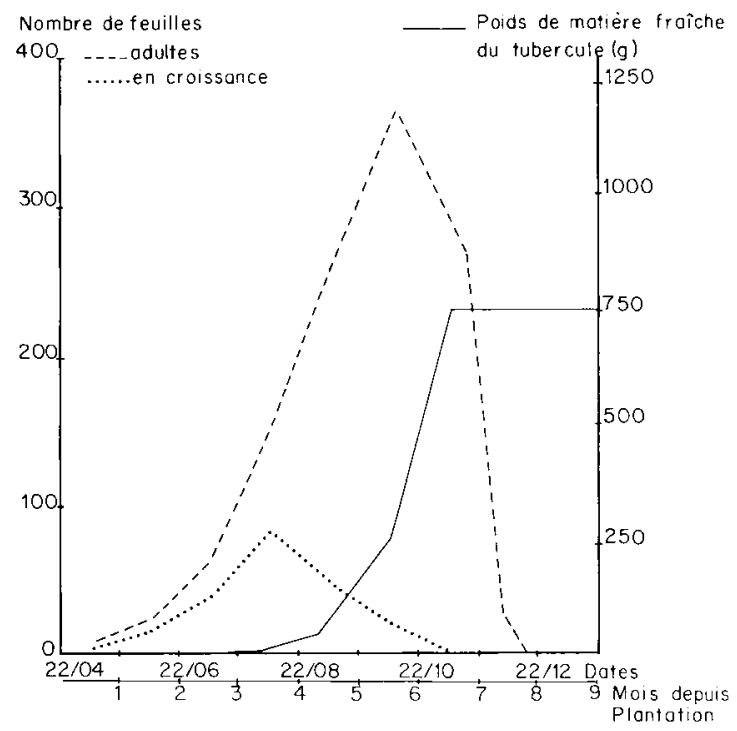

Figure 10

Evolutions comparées de la partie aérienne et de la partie souterraine.

Comparison of growth of aerial and underground parts.

..... - Number of full-grown leaves.

...... Number of growing leaves.

Tuber dry matter weight.

TABLEAU 2

Croissance et développement de D. alata $c v$. "Lupias " planté en avril :

caractéristiques comparées des plants obtenus par germination de fragments de tubercules (CLAIRON \& ZINSOU, 1980) et des vitroplants.

Growth and development of D. alata cv. "Lupias" planted in April :

comparison of plants obtained by germinating tuber fragments (CLAIRON \& ZINSOU, 1980) and vitroplants.

\begin{tabular}{|c|c|c|c|}
\hline & Caractère & $\begin{array}{l}\text { Plante issue d'un fragment } \\
\text { de tubercule-mère } \\
\text { de } 150 \text { à } 250 \mathrm{~g}\end{array}$ & Vitroplant \\
\hline \multirow{5}{*}{$\begin{array}{l}\text { Partie aérienne } \\
\text { Aerial part }\end{array}$} & Poids de matière sèche à 2 mois & $23 \mathrm{~g}$ & $7 \mathrm{~g}$ \\
\hline & Poids maximal de matière sèche & $260 \mathrm{~g}$ & $96 \mathrm{~g}$ \\
\hline & Délai de croissance aérienne maximale & 6 mois & 6 mois \\
\hline & $\begin{array}{l}\text { Durée de la phase feuillée (depuis plan- } \\
\text { tation) }\end{array}$ & 8 mois & 8 mois \\
\hline & $\begin{array}{l}\text { Nombre de tiges issues de la base de la } \\
\text { plante }\end{array}$ & 2 , issues du collet & $\begin{array}{l}4 \text {, issues du collet }+11 \text { axillaires } \\
\text { basaux }\end{array}$ \\
\hline \multirow{5}{*}{$\begin{array}{l}\text { Partie souterraine } \\
\text { Underground part }\end{array}$} & Nombre de prétubercules & 2 & $\geq 4$ \\
\hline & Nombre de tubercules & 2 & 2,5 \\
\hline & $\begin{array}{l}\text { Poids de matière sèche des tubercules } \\
\text { à } 4 \text { mois }\end{array}$ & $30 \mathrm{~g}$ & $8 \mathrm{~g}$ \\
\hline & Poids de matière sèche à la récolte & $600 \mathrm{~g}$ & $250 \mathrm{~g}$ \\
\hline & Teneur en matière sèche des tubercules & $\begin{array}{l}\text { Saut de } 25,5 \% \text { à } 32,8 \% \text { entre } \\
\text { octobre et novembre, varie peu } \\
\text { ensuite }\end{array}$ & $\begin{array}{l}\text { Saut de } 25 \% \text { à } 33 \% \text { entre octobre } \\
\text { et novembre ; varie peu ensuite }\end{array}$ \\
\hline
\end{tabular}

Durée du repos végétatif (dessèchement du feuillage - ger- 


\section{B. Aspects agronomiques}

Notre expérimentation montre clairement la possibilité de mener à son terme une culture au champ de vitroplants d'igname. Les rendements obtenus sont environ la moitié de ceux d'une culture traditionnelle du même cultivar "Lupias" (CLAIRON \& ZINSOU, 1980). Cependant, la variabilité individuelle laisse penser qu'une sélection plus sévère au cours de la culture in vitro serait susceptible d'améliorer le rendement moyen par plante ; en outre, on peut envisager d'augmenter le nombre de plantes par $\mathrm{m}^{2}$ pour atteindre un rendement à l'hectare équivalent à celui des cultures traditionnelles. Il faut de toutes façons être prudent dans une évaluation agronomique quantitative vu l'effectif limité dont nous disposons (57 plantes) et la surface expérimentale très inférieure à celle d'une plantation professionnelle. Nos résultats ne s'appliquent en outre qu'à une situation agronomique particulière (conditions pédoclimatiques et itinéraire technique). Malgré ces réserves, la possibilité de prévision du rendement 3 mois avant la nécrose totale de la partie aérienne est certainement généralisable. Il en est très probablement de même pour des remarques concernant la notion de maturité.
Alors que la nécrose totale de l'appareil aérien ne survient que fin décembre, le maximum du poids de matière sèche et le maximum de la teneur en matière sèche sont atteints dès novembre. Les teneurs en glucides étant fixes depuis longtemps (LACOINTE, 1984), on peut donc récolter le tubercule à ce stade sans conséquence sur le rendement.

La "maturité " ainsi définie paraît avoir une signification plus physiologique. En effet, les tubercules récoltés à partir de novembre germent fin avril, en même temps que ceux récoltés après nécrose totale de la partie aérienne, alors que ceux récoltés en septembre et octobre germent significativement plus tôt, fin mars.

Reçu le 25 septembre 1986. Accepté le 5 février 1987.

\section{REMERCIEMENTS}

Nous remercions R. BonHomme (Station de Bioclimatologie) pour sa contribution à l'estimation du rendement de la transformation de l'énergie lumineuse incidente en matière sèche stockée.

\section{RÉFÉRENCES BIBLIOGRAPHIQUES}

Ahoussou N., Touré B., Piquepaille P., 1980. Etude de la variabilité créée par la nature de l'organe de multiplication végétative chez Dioscorea alata L. cv. Brazo fuerté. Communication in : "L'igname ", Séminaire Intern., Pointe-à-Pitre, Guadeloupe, 28 juill.-2 août 1980, I.N.R.A., Paris, 191-205.

Arnolin R., Degras L., 1983. Résultats et perspectives en culture in vitro chez les ignames alimentaires (in vitro culture, results and prospects for food yams). Communication, VIth Symposium Intern. Soc. Trop. Root Crops, 1984. p. 641-648, édit. CIP, Lima, Pérou.

Chapman T., 1965. Some investigations into factors limiting yields of the White Lisbon Yam (Dioscorea alata L.) under Trinidad conditions. Trop. Agric. (Trinidad), 42, 145-171.

Chaturvedi H. C., 1975. Propagation of Dioscorea floribunda from in vitro culture of single-node stem segments. Curr. Sci., 44 (23), 839-841.

Clairon M., Zinsou C., 1980. Etude de plantations échelonnées d'Igname (Dioscorea alata, cv. "Lupias ») : effet du vieillissement du tubercule sur la croissance et le développement de la plante. Communication, in : "L'igname », Séminaire Intern., Pointe-àPitre, Guadeloupe, 28 juill.-2 août 1980, I.N.R.A., Paris, 125-141.

Dagnélie P., 1975a. Théorie et méthodes statistiques. Applications agronomiques. Vol. 2. Les méthodes de l'inférence statistique. Les Presses Agronomiques de Gembloux, Vander, Bruxelles, $463 \mathrm{p}$

Dagnélie P., 1975b. Analyse statistique à plusieurs variables. Les Presses Agronomiques de Gembloux, Vander, Bruxelles, 362 p.

Degras L., Arnolin R., Poitout A., Suard C., 1977a. Quelques aspects de la biologie des Ignames (Dioscorea spp.). I. Les ignames et leur culture. Ann. Am. Plantes, 27, 1-23.

Degras L., Vautor A., Poitout R., Arnolin R., Suard C., $1977 b$. Croissance et développement de l'igname Cousse-Couche (Dioscorea trifida L.). Communication, XIVth Annual Meeting CFCS Guadeloupe et Martinique, 27 juin-2 juillet 1977. 6 p.

Degras L., Poitout R., Suard C., Vautor A., 1977c. Croissance et développement de l'igname Dioscorea alata. Communication, XIVth Annual Meeting CFCS, Guadeloupe et Martinique, 27 juin2 juillet 1977. $5 \mathrm{p}$.

Degras L., 1978. La reproduction végétative de l'igname. Données fondamentales et applications récentes. Communication, Séminaire International sur I'Igname, Buéa, Cameroun, 1-8 octobre 1978, IFS provisional report $n^{\circ} 3,93-121$.
Lacointe A., 1984. Quelques aspects du comportement d'une plante tubérifère tropicale (igname) issue de culture in vitro, en plantations échelonnées. Thèse de $3^{\mathrm{e}}$ cycle, Univ. Clermont-Ferrand II, $73 \mathrm{p}$.

Lawton J. R., Lawton J. R. S., 1969. The development of the tuber in seedlings of five species of Dioscorea from Nigeria. Bot. J. Linn. Soc., 62, 223-232.

Mantell S. H., Haque S. Q., 1979. Disease-free yams : their production, maintenance and performance. Project bulletin $n^{\circ} 2$.

Mascarenhas A. F., Hendre R. R., Nadgir A. L., Ghugale D. D., Godbole D. A., Prabhu R. A., Jagannathan V., 1976. Development of plantlets from cultured tissues of Dioscorea deltoidea Wall. Ind. J. exp. Biol., 14, 604-606.

Mathurin P., 1983. Comportement, production et intérêt des plantes sevrées issues de tubercules entiers ou fragmentés d'igname $D$. alata cv. Pacala. Ann. Univ. Abidjan, Série C (Sciences), XIX, 239. 260.

Miège J., 1952. Contribution à l'étude systématique des Dioscorea d'Afrique occidentale. Thèse Sc. Nat., Paris, $266 \mathrm{p}$.

Miège J., 1957. Influence de quelques caractères des tuberculessemences sur la levée et le rendement des ignames cultivées. $J$. Agric. trop. Bot. appl., 4, 315-342.

Morel G., Martin C., 1955. Guérison de pommes de terre atteintes de maladie à virus. C. R. Acad. Agric. Fr., 41, 472-475.

Murashige T., Skoog F., 1962. A revised medium for rapid growth and bioassays with tobacco tissue cultures. Physiol. Plant., 15, 473497.

Okoli O. O., 1980. Dry matter accumulation and tuber sprouting in yams (Dioscorea spp.). Exp. Agric., 16, 161-167.

Sadik S., Okereke O. U., 1975. Flowering, pollen grain germination, fruiting, seed germination and seedling development on white yam, Dioscorea rotundata Poir. Ann. Bot., 39, 597-604.

Sobulo R. A., 1972. Studies on white yam (Dioscorea rotundata) 1. Growth analysis. Exp. Agric., 8, 99-106

Trouslot M. F., 1978. Croissance et tubérisation chez quelques cvs de Dioscorea cayenensis Lamk. Communication, Séminaire International sur l'igname, Buéa, Cameroun, IFS provisional report $n^{\circ} 3$, 153-182.

Trouslot M. F., 1985. Analyse de la croissance et de la morphogenèse de l'igname Dioscorea complexe D. cayenensis-D. rotundata Thèse d'Etat, Univ. Clermont-Ferrand II. Collection : «Travaux et Documents ", ed. ORSTOM, n ${ }^{\circ} 185,370 \mathrm{p}$. 